bioRxiv preprint doi: https://doi org/10.1101/2021.05.21.445141. this version posted May 23, 2021. The copyright holder for this preprint

(which was not certified by peer review) is the author/funder, who has granted bioRxiv a license to display the preprint in perpetuity. It is made available under aCC-BY 4.0 International license.

\title{
Silencing Doublesex expression triggers three-level pheromonal feminization in Nasonia males
}

Yidong Wanga, Weizhao Suna,d, Sonja Fleischmann, Jocelyn G. Millarc, Joachim Rutherb, ${ }^{\text {*} E v e l i n e ~ C ~}$ Verhulsta

a. Wageningen University, Laboratory of Entomology, Wageningen, The Netherlands

b. University of Regensburg, Institute of Zoology, Regensburg, Germany

c. University of California Riverside, Department of Entomology, Riverside, CA, United States

d. Current address: University of Münster, Institute for Evolution \& Biodiversity, Münster, Germany

* corresponding author: Eveline C Verhulst 


\section{Abstract}

The transcription factor Doublesex (Dsx) has a conserved function in controlling sexual morphological differences in insects, but our knowledge on its role in regulating sexual behavior is widely limited to Drosophila. Here, we show in the parasitoid wasp Nasonia vitripennis that males whose Dsx gene had been silenced by RNA interference (NvDsx-i) underwent a three-level pheromonal feminization: (1) $N v D s x$-i males were no longer able to attract females from a distance, owing to drastically reduced titers of the abdominal long-range sex pheromone. (2) NvDsx-i males were courted by wild-type males like females which correlated with a lower abundance of alkenes in their cuticular hydrocarbon (CHC) profiles. Supplementation of $N v D s x-i$ male $\mathrm{CHC}$ profiles with realistic amounts of synthetic (Z)-9hentriacontene (Z9C31), the most significantly reduced alkene in $N v D s x$-i males, interrupted courtship by wild-type conspecific males. Supplementation of female $\mathrm{CHC}$ profiles with $\mathrm{Z9C} 31$ reduced courtship and mating attempts by wild-type males. These results prove that $Z 9 C 31$ is crucial for sex discrimination in Nasonia. (3) Nvdsx-i males were hampered in eliciting female receptivity during courtship and thus experienced severely reduced mating success, suggesting that they are unable to produce the hitherto unidentified oral aphrodisiac pheromone reported in $N$. vitripennis males. We conclude that Dsx is a multi-level key regulator of pheromone-mediated sexual communication in N. vitripennis. Silencing Dsx by RNA interference provides a new avenue for unraveling the molecular mechanisms underlying the pheromone-mediated sexual communication in insects. 


\section{Introduction}

Insect sex pheromones play a major role in initiating and guiding the mating process, and in allowing individuals to reliably recognize conspecific mates. In many insect species one sex releases a blend of volatile sex pheromones that transmits a species-specific message to attract conspecifics of the opposite sex (1-3). The attractiveness of the blend is often based on the quantity and ratio of the pheromone components, which may be correlated with nutritional condition, age, or gamete quality of the sender $(4,5)$. Over shorter distances, sex-specific mixtures of low-volatility, fatty-acid-derived hydrocarbons on the insect cuticle (cuticular hydrocarbons, $\mathrm{CHC}$ ) play a role in the recognition and mating processes of many insect species (6-10), and species can often be identified by their unique $\mathrm{CHC}$ profiles $(6,11,12)$. In many insects, the same $\mathrm{CHC}$ compounds are shared by both sexes but vary in their relative abundance (13-15), while others have evolved subsets of sex-specific $\mathrm{CHC}$ to either attract potential mates or to repel competitors $(16,17)$.

Because sex pheromone production is sexually dimorphic in nature, it is generally assumed that the sex determination pathway is involved in regulating sex pheromone production. In insects, sexual development is initiated by diverse species-specific primary signals, but downstream, the pathway contains several transcription factors that have relatively conserved functions $(18,19)$. One major transcription factor, Doublesex (Dsx), is located at the bottom of the cascade, and regulates sexual differentiation in various species across insect orders (20-26). At first, Dsx was considered to be exclusively a regulator of sex-specific morphological development, while the transcription factor Fruitless (Fru), as identified in Drosophila melanogaster, was thought to mediate sex-specific behaviours, including mating behaviour. In Drosophila, the male Fru protein $\left(\mathrm{Fru}^{\mathrm{M}}\right)$ is a key regulator of crucial mating rituals in both the central nervous system (CNS) and the peripheral nervous system $(27,28)$. Accumulating evidence has been shown that Dsx coordinates the development of the CNS, and together with FruM, shapes courtship behaviour in D. melanogaster males (29-31). Nevertheless, Dsx knockdown experiments in other insect species, for example in Onthophagus taurus beetles, changed aggressiveness, but did not yield solid evidence for changes in mating behaviours (32). Our knowledge of the role of Dsx in directly regulating the mating behaviour of insects is therefore primarily limited to Drosophila flies. 
Mating is a complex process in insects, which is influenced by morphological characteristics, behaviour, and chemical communication, making it a challenge to study the role of Dsx in regulating these interacting factors. The jewel wasp Nasonia vitripennis (Hymenoptera: Pteromalidae), as an emerging model species, offers great opportunities for this kind of research. Nasonia vitripennis is a gregarious parasitoid of several pest fly species (33). The sex determination mechanism in N. vitripennis has been completely elucidated (34-37), and recently we illustrated the role of Dsx in regulating sexually dimorphic traits (38). In parallel, the chemical communication system of $N$. vitripennis has been intensively studied (39). The male-derived long-range sex attractant pheromone of $N$. vitripennis consists of the major components $(4 R, 5 R)$ - and $(4 R, 5 S)$-5-hydroxy-4-decanolide (HDL-RR and HDL$R S)$ with a synergistic minor compound, 4-methylquinazoline (4MQ) $(40,41)$. The pheromone is synthesized by males in the rectal vesicle (42) and is deposited onto the natal host and other substrates through a typical "abdominal dipping" behaviour $(43,44)$. Pheromone marks are highly attractive to conspecific virgin females, whereas mated females are no longer attracted (45). In addition to the malederived sex attractant, sex- and species-specific $\mathrm{CHC}$ profiles have been identified in wasps of the genus Nasonia $(6,13,46,47)$. CHC are used for mate recognition, and elicit stereotyped courtship behaviour in $N$. vitripennis males which has been well-characterized and described in detail (48-50). After encountering a female, the male immediately mounts, places his fore tarsi on her head, and starts to perform the mating ritual, which includes stereotypical wing movements, a series of head nods, and antennal sweeping (48). During head nodding, the male moves his mouthparts close to the proximal region of the female's antennae and synchronizes the extrusion and retraction of his mouthparts with the upward and downward nodding process $(48,49)$. During this crucial step, males are believed to secrete an aphrodisiac pheromone from the mouthparts onto the female antennae to elicit female receptivity (50). The female signals receptivity by lowering her antennae while raising her abdomen to expose her genital orifice, after which mating usually occurs. Subsequently, another short round of courting, termed post-copulatory courtship, is repeated by the male, during which the female often again exhibits a receptive posture. The sequence terminates with the male dismounting from the female. The blend of oral pheromones apparently not only serves as an aphrodisiac, but also inhibits the response of mated females to the male sex pheromone, thus lowering the chance of re-mating (39). Taken together, many aspects of the Nasonia mating behaviour and chemical signaling are known, but the possible role of $D s x$ in regulating these sex- and species-specific traits has not yet been investigated. 
In this research, we explored the role of $N$. vitripennis $D s x(N v D s x)$ in regulating male mating behaviour and sex pheromone production. We silenced $N v D s x$ in male pupae by RNA interference (NvDsx-i) and observed each step of the resulting adults' mating behaviour after emergence. Using coupled gas chromatography-mass spectrometry (GC-MS), we then investigated the effects of NvDsx on the pheromone chemistry by comparing long-range sex pheromone titers and $\mathrm{CHC}$ profiles of $N v D s x-\mathrm{i}$ males with those of green fluorescent protein RNA interference mock control males (gfp-i). Finally, we performed Y-tube olfactometer bioassays and bioassays with treated dummies to determine the attractiveness of $N v D s x-i$ males to virgin females with which we could identify key components of the male $\mathrm{CHC}$ that inhibit male-male mating attempts.

\section{Results}

\section{Courting NvDsx-i males are less successful in rendering conspecific females receptive.}

We first asked if the silencing of NvDsx expression affects the mating behaviour of males. We microinjected Dsx dsRNA into white male pupae and measured a significant suppression of NvDsx in these $N v D s x$-i males after emergence when compared to the gfp-i control males. (GLM, $P<0.001$ ), or to Non-injected males (Non-i, GLM, $P<0.001$ ) (Fig. 1a). In mating trials, we exposed virgin females to $N v D s x-i, g f p-i$, or Non-i males and recorded the duration of the behavioural elements searching, mounting including head nodding, copulation, and post-copulatory courtship, as well as male mating success rate. Only $10.9 \%$ of $N v D s x$-i males elicited receptivity and subsequently mated, which was significantly less than Non-i (97.6\%) and gfp-i males (100\%, Fig. 1b). NvDsx-i males did not differ from Non-i (GLM, $P=0.940)$ and $g f p-i(G L M, P=0.951)$ males with respect to searching times, but the high fraction of unreceptive females led to a significantly longer mounting time for NvDsx-i males (Non-i: GLM, $P<0.001$; gfp-i: GLM, $P<0.001$, Fig. 1c). The few $N v D s x-i$ males that elicit female receptivity after mounting showed no significant difference in copulation time (Non-i: GLM, P=0.540; gfp-i: GLM, $P=0.266$ ), but displayed significantly shorter post-copulatory courtship than Non-i males (GLM, $P<0.001$ ) and $g f p-i$ males (GLM, $P=0.002$ ). There was an effect of $g f p$ RNAi on the courtship behaviour, as $g f p-i$ males showed longer mounting time (GLM, P<0.001) and shorter post-copulatory courtship (GLM, $P=0.014$ ) than Non-i males. However, the $100 \%$ mating success rate of $g f p-i$ males indicates overall mating performance was unaffected. 


\section{$N v D s x-i$ males are no longer attractive to virgin females due to decreased abdominal sex attractant pheromone production.}

To evaluate whether NvDsx-i males were less successful in attracting virgin females from a distance, we gave virgin females the choice between the odours of two groups of ten live conspecifics in a Y-tube olfactometer. As expected, virgin females preferred the odour of Non-i males (GLM, $P<0.01$, Fig. 2a) or gfp-i males (GLM, $P<0.001)$ over that of Non-i females. The attractiveness of Non-i and gfp-i males did not differ in these trials (GLM, $P=0.904)$. In contrast, virgin females were not attracted by the odours of NvDsx-i males, showing no preference between those odours and the odours of Non-i females (GLM, $P=0.856$ ). When given the choice, virgin females did not discriminate between the odours of Non-i males and gfp-i males but they preferred Non-i males over NvDsx-i males (GLM, $P<0.001$, Fig. 2a). The reduced attractiveness of $N v D s x$-i males in comparison to gfp-i males correlated with significantly reduced titres of the abdominal sex attractant pheromones, as determined by GC-MS (Fig. 2b). All three abdominal sex attractant pheromone components, HDL-RR, HDL-RS, and $4 \mathrm{MQ}$, were detected only in trace amounts in extracts of the abdomens of $N v D s x-i$ males, while the total amount and relative proportions of abdominal sex attractant pheromone components in gfp-i males were within the range reported previously for Non-i males $(41,51,52)$ (Fig. 2c-d).

$N v D s x-i$ males elicit courtship in conspecific males due to decreased alkene abundance in their CHC profiles.

Earlier observations of $N v D s x-i$ mating behaviour by our group suggested that $N v D s x-i$ males are mistaken for females by normal males. Previous research also showed that males use $\mathrm{CHC}$ signaling to discriminate between the sexes, and that female-derived $\mathrm{CHC}$ arrests males and elicits courtship behaviour (13). Hence, we hypothesized that the unusual courting of $N v D s x$-i males by Non-i males is elicited by changes in their $\mathrm{CHC}$ profiles. One characteristic feature of $N$. vitripennis $\mathrm{CHC}$ profiles is the male-biased relative abundance of alkenes (13). Analysis of the $\mathrm{CHC}$ profiles of $N v D s x-i$ and gfp-i males indeed revealed a significant reduction in alkenes (Fig. 3a-b). The amounts of (Z)-9-hentriacontene (Z9C31) in CHC extracts from NvDsx-i males were less than one-fourth of those found in gfp-i males (mean \pm SE gfp-i: $31.8 \pm 5.7 \mathrm{ng} ; N v D s x$-i: $6.6 \pm 1.1 \mathrm{ng}$ ). Similar reductions, albeit to a lesser extent, were found for (Z)-7-hentriacontene (Z7C31), (Z)-9-tritriacontene (Z9C33), and (Z)-7-tritriacontene (Z7C33). 
These results suggest that the decrease in alkenes explains the same-sex courtship of $N v D s x$-i males. Given that $N v D s x-i$ treatment affected the amounts of $Z 9 C 31$ the most, we tested whether the application of natural amounts of this compound to NvDsx-i males was sufficient to interrupt this male-male courtship. Nasonia males are known to respond to attractive dead conspecifics by mounting and copulation attempts (50). Therefore, we conducted a series of behavioural experiments with differently treated dead wasps (dummies). We presented Non-i males with untreated gfp-i and NvDsx-i dummies as well as with those treated with $30 \mathrm{ng}$ of synthetic Z9C31 dissolved in dichloromethane (DCM) or the pure DCM solvent (control). In general, a higher percentage of $N$. vitripennis Non-i males tried to copulate with $N v D s X-i$ male dummies than with gfp-i male dummies $\left(X^{2}(1, N=50)=8, P=0.028\right.$, Fig. 4a), spent significantly more time on NvDsx-i male dummies than on gfp-i male dummies (Mann-Whitney $U=149, n_{1}=n_{2}=25, P<0.01$, Fig. $4 \mathrm{~b}$ ), and tried to copulate with $N v D s x$-i male dummies longer than with gfp-i male dummies (Mann-Whitney $U=209, n_{1}=n_{2}=25, P=0.012$, Fig. 4c). The application of pure DCM to $N v D s x$-i male dummies did not significantly alter the courtship behaviour of Non-i males (Fig. 4a-c). However, comparing to the pure DCM solvent treated NvDsx-i male dummies, the application of Z9C31 to $N v D s x$-i male dummies significantly reduced the proportion of Non-i males attempting to copulate with those dummies $\left(X^{2}(1, N=50)=20.053, P<0.001\right)$, the duration of mounting (Mann-Whitney $U=46.5$, $n_{1}=n_{2}=25, P<0.001$ ) and copulation attempts (Mann-Whitney $U=130, n_{1}=n_{2}=25, P<0.01$ ), to the levels of gfp-i dummies (Fig. 4a-c). These results support our hypothesis that males use the increased abundance of alkenes in the $\mathrm{CHC}$ profiles of males to discriminate between sexes. Therefore, in the next step we investigated whether treatment with $29 \mathrm{C} 31$ would make female dummies less attractive to responding males. To this end, we performed a similar dummy experiment as described above, using Non-i male (negative control) and differently treated Non-i female dummies. A significantly higher proportion of $N$. vitripennis Non-i males tried to copulate with Non-i female dummies than with Non-i male dummies $\left(X^{2}(1, N=40)=28.972, P<0.001\right)$, and Non-i males mounted (Mann-Whitney $U=22$, $n_{1}=n_{2}=20, P<0.001$ ) and tried to copulate (Mann-Whitney $U=21, n_{1}=n_{2}=20, P<0.001$ ) significantly longer with Non-i female dummies than with Non-i male dummies (Fig. 4d-f). Application of pure DCM solvent to Non-i female dummies did not influence the response of Non-i males. However, comparing to the pure DCM solvent treated Non-i female dummies, application of $30 \mathrm{ng}$ of Z9C31 to Non-i female dummies reduced the proportion of mating attempts by Non-i males $\left(X^{2}(1, N=40)=13.333, P<0.01\right)$ as well as the total time Non-i males spent trying to copulate with the Non-i female dummies to intermediate 
levels (Mann-Whitney $U=59, n_{1}=n_{2}=20, P<0.001$, Fig. 4 d,f). The duration of mounting was also reduced and was not significantly different than that of Non-i male dummies (Mann-Whitney $U=121, n_{1}=n_{2}=20$, $P=0.202$, Fig. 4e).

\section{Discussion}

In this study we set out to identify the role of Dsx in regulating N. vitripennis male mating behaviour and pheromone production, and we found a major effect of NvDsx in the regulation of the production of both volatile and non-volatile sex pheromones. Using Y-olfactometer two-choice assays and GC-MS analyses, we observed a reduced attractiveness of $N v D s x$-i males to virgin females accompanied by the almost complete disappearance of the abdominal volatile sex attractant pheromone components $\mathrm{HDL}-R R$, HDL-RS, and 4MQ. HDL-RR and HDL-RS are synthesized by $N$. vitripennis males in the rectal vesicle from fatty acids, and are released via the anal orifice (42). Our results suggest that NvDsx directly regulates the biosynthetic pathway, but which of the genes involved in the biosynthesis of HDL (53) are targeted by NvDsx, remains unknown. Although it has been suggested that the production of the $\mathrm{CHC}$ pheromones of females is controlled by Dsx in $D$. melanogaster $(54,55)$, hardly any research has focused on this topic in other insects. Hence, the present study is, to our knowledge, the first to demonstrate a regulatory effect of Dsx on the biosynthesis of pheromones other than $\mathrm{CHC}$ in insects.

Despite their lack of abdominal sex attractant pheromones for long-range attraction of females, all tested NvDsx-i males were still able to mount and court females, and displayed stereotypical mating behaviours such as head nodding and antennal sweeping. Apparently, the substantial reduction in the titres of the sex attractant pheromone did not affect the acceptance of courtship by females, initiated by the males through mounting. We also found no significant differences in the searching times for the different treatments in the mating experiment, which may have been due to the experimental set-up, in which the male and female were enclosed in a small space. At this level of the mate finding process, the abdominal sex attractant pheromone of $N$. vitripennis males no longer plays a role, and it is up to the male to recognize and court the female via the female-specific CHC (13). Intriguingly, NvDsx-i males were courted by Non-i males in a similar manner to females, suggesting a kind of "feminization" of the NvDsxi CHC profiles. NvDsx-i males apparently became sexually attractive to Non-i males because of a substantial reduction of alkenes, in particular Z9C31, in their CHC profile. Supplementation of the profile by applying synthetic $Z 9 C 31$ to $N v D s x-i$ male dummies eliminated the courting behaviour of Non-i males, 
suggesting that $Z 9 \mathrm{C} 31$ is a key compound for sex discrimination by $N$. vitripennis males. Just as the cuticular pheromone (Z)-7-tricosene inhibits male-male courtship in D. melanogaster (16, 56), Z9C31 helps to prevent male-male courtship in N. vitripennis. The low abundance of Z9C31 in the CHC profile of females (13) might even underlie males' recognition of potential mates. This idea is supported by our finding that applying $29 \mathrm{C} 31$ to female dummies reduced Non-i male courting attempts and duration, although not to the low levels shown towards Non-i males. In this context, it is important to note that the other three alkenes, and many other $\mathrm{CHC}$ components that are found in different relative amounts in $\mathrm{N}$. vitripennis males and females might also contribute to this effect $(13,57)$. Hence, the addition of $Z 9 C 31$ alone might not be sufficient to entirely disrupt a female's pheromone signal.

We analyzed the presence and amounts of alkenes that we found to be directly or indirectly regulated by NvDsx in the previously published, species-specific $\mathrm{CHC}$ profiles of Nasonia (6). We found a male bias in the expression of these alkenes in N. vitripennis but not in the other three Nasonia species (6, 47). In D. melanogaster, alkenes are a major fraction of the CHC profiles (58), and their biosynthesis requires different types of desaturases (59). Niehuis et al. (2011) found that two of the Drosophila desaturase gene homologs map to regions in the Nasonia genome that contain the QTL clusters for these alkenes. NvDsx is a prime candidate for regulation of the expression of the desaturase genes that controls alkene biosynthesis. The gain and loss of the binding-site of Dsx in these desaturase genes may account for the species-specific differences in alkene synthesis, as has been shown for $D$. melanogaster (60). This difference might be related to specific life history traits of different Nasonia species. Females mating inside the host puparium (within-host mating, WHM) is rare in parasitoids, but it does happen in Nasonia (61). Compared to N. vitripennis, the sibling species $N$. giraulti and $N$. longicornis show a significantly higher WHM rate (61). Because $N$. vitripennis is ancestral to the other species in the genus (62), this sex-specific difference in alkene expression might have been lost in $N$. giraulti and $\mathrm{N}$. longicornis as a result of decreased mate competition due to their higher WHM rates. Further research will be required to verify this. In addition, behavioural assays to determine mate discrimination among Muscidifurax uniraptor, N. vitripennis, and Trichomalopsis sarcophagae showed that $N$. vitripennis males do not discriminate against $T$. sarcophagae females, displaying courtship behaviour and copulation attempts, but reject $M$. uniraptor females (46). Despite the general similarity of the $\mathrm{CHC}$ profiles of females of both species, both $Z 9 \mathrm{C} 31$ and $Z 9 \mathrm{C} 33$ are produced by $N$. vitripennis and $T$. sarcophagae females at low levels, whereas M. uniraptor females have higher amounts of these 
alkenes in their $\mathrm{CHC}$ profiles (46). Hence, Z9C31 and the other alkenes might also be involved in species discrimination by $N$. vitripennis males. It would be interesting to further investigate the consequences and benefits of maintaining this alkene-dependent olfactory recognition system.

By comparing the courtship and copulation behaviours of NvDsx-i, gfp-i, and Non-i males when presented with live virgin females, we did not find obvious differences in male courtship performance between NvDsx-i and Non-i males. Male courtship behaviour in insects is thought to be governed primarily by Fruitless (Fru) (28). However, in Drosophila, Dsx was shown to facilitate the formation of the CNS, and these neuronal networks develop during pupation (63). This suggests that silencing NvDsx from the pupal stage onwards does not affect the development of the regions of the CNS that control mating behaviour in $N$. vitripennis males. Hence, our results add to the current theory that Fru is the main regulator of courtship behaviour in insects, but it should be noted that silencing $N v D s x$ earlier in development might produce different outcomes.

We observed significantly prolonged mounting time and a strong reduction in mating success of NvDsxi males when they courted normal females, indicating that an important component inducing receptivity of females might be missing from the NvDsx-i males. It has been shown that males with sealed mouthparts cannot induce receptivity in females, which indicates the existence of an aphrodisiac produced by the mouthparts $(50,64)$. The identity of this aphrodisiac is still unknown despite extensive efforts to unravel its chemical structure (39). So far, three fatty acid ethyl esters have been identified in the oral secretion of $N$. vitripennis males, but these compounds were unable to elicit female receptivity (65). The inability of $N v D s x-i$ males to elicit receptivity in conspecific females suggests that the production of the oral aphrodisiac is affected by Dsx, possibly in combination with, or due to malformed mandibular glands, which are known to be present in $N$. vitripennis (66). Therefore, comparative chemical analyses of the heads of NvDsx-i and Non-i males will be a promising approach to unravel the identity of the oral aphrodisiac, as a next step towards a comprehensive understanding of pheromone communication in N. vitripennis.

\section{Material and methods}

1. Insect rearing 
The Wolbachia-free AsymCx laboratory strain of N. vitripennis was used in all experiments. The laboratory cultures were constantly reared on the host Calliphora sp. and Lucilia caesar (only the wasp for dead wasp bioassay) at $25^{\circ} \mathrm{C}$ with a $16 \mathrm{~h} / 8 \mathrm{~h}$ light/dark cycle.

\section{NvDsx dsRNA preparation}

NvDsx knockdown was conducted by using RNA interference (RNAi) as described in Wang et al. (2020). MEGAscript RNAi Kit (Invitrogen ${ }^{\mathrm{TM}}$, Waltham, Massachusetts, USA) was used to produce the dsRNA that targets the common region (exons 2-5) of $N v D s x$ in all male splice-variants. Gfp dsRNA was used as a control for all experiments and was generated from the vector pOPINEneo-3C-GFP (Addgene plasmid \# 53534; http://n2t.net/addgene: 53534; RRID: Addgene_53534). Primers that were used to construct the dsRNA T7 template were designed in Geneious 10.0.9 (Table S1). Both NvDsx and gfp dsRNAs were diluted in RNase-free water to a final concentration of $4000 \mathrm{ng} / \mathrm{ul}$ (determined using a NanoDrop ${ }^{\mathrm{TM}} 2000$ Spectrophotometer, Thermo Scientific ${ }^{\mathrm{TM}}$ ) and stored at $-20^{\circ} \mathrm{C}$.

\section{Microinjection}

DsRNAs were pre-mixed with a prepared red food dye at a 9:1 ratio before microinjection. Commercially available red food dye was prepared by diluting it $2 \mathrm{X}$ in DNase/RNase free water. Because asexual reproduction leads to all-male broods in the haplodiploid sex determination system of Hymenoptera, virgin females were used to produce the male offspring that were used at the pupal stage for microinjection. Thirty female pupae were collected in the black pupal stage ( 12 days after egg laying), transferred to a single glass vial $(7.5 \mathrm{~cm}$ length, one $\mathrm{cm}$ diameter), plugged with a cotton plug, and kept under rearing conditions as described above. Twenty-four hours after eclosion, each female was provided with two Calliphora sp. host pupae per day. Host pupae were continuously incubated under rearing conditions until parasitoid offspring reached the white pupal stage ( $\sim 7$ days after egg laying). Pupae of male $N$. vitripennis were subsequently collected from hosts and fixed on glass slides with double-sided adhesive tape (3M). Microinjection was performed with a FemtoJet ${ }^{\circledR} 4 \mathrm{i}$ microinjector (Eppendorf, Hamburg, Germany) following the protocol described by Lynch and Desplan (2006). Phosphate Buffered Saline (PBS) plates were prepared by adding $1.5 \mathrm{~g}$ BD Bacto ${ }^{\mathrm{TM}}$ Agar (Fisher Scientific, Sparks, Nevada, USA) and one Oxoid ${ }^{\mathrm{TM}}$ phosphate buffered saline tablet (Thermo Scientific ${ }^{\mathrm{TM}}, \mathrm{USA}$ ) into $100 \mathrm{ml}$ distilled water and autoclaving at $115^{\circ} \mathrm{C}$ for 15 minutes before pouring 
them into plastic petri-dishes. After microinjection, slides with injected pupae were placed on PBS plates to prevent dehydration and incubated under rearing conditions until adult emergence.

\section{Quantification of NvDsx expression by qPCR}

NvDsx-i males were collected once they emerged. Six individuals were pooled as one biological replicate and six biological replicates per treatment were collected in $1.5 \mathrm{ml}$ microcentrifuge tubes and flash frozen in liquid nitrogen. ZR Tissue \& Insect RNA MicroPrep ${ }^{\mathrm{TM}}$ (Zymo Research Corp., Irvine, California, USA) kit was used under the manufacturer's instructions to extract the total RNA with an oncolumn DNase treatment step. Sixteen $\mu$ l of DNase/RNase free water was applied to elute each sample. RNA concentration was determined with a NanoDrop ${ }^{\text {TM }} 2000$ Spectrophotometer (Thermo Scientific ${ }^{\mathrm{TM}}$ ) and one $\mu \mathrm{g}$ of total RNA from each sample was converted into cDNA with SensiFAST ${ }^{\mathrm{TM}}$ CDNA Synthesis Kit (Bioline, London, UK) using a T100 TM Thermal Cycler (Bio-Rad) with an incubation program consisting of five minutes priming at $25^{\circ} \mathrm{C}, 30$ minutes at $46^{\circ} \mathrm{C}$ and five minutes at $85^{\circ} \mathrm{C}$.

Following the manufacturer's instruction, a SensiFAST ${ }^{\mathrm{TM}} \mathrm{SYBR}^{\circledR}$ No-ROX Kit (Bioline, London, UK) was used to perform the qPCR. cDNA templates were further diluted 1:100 and five $\mu$ l aliquots of each diluted template were used in qPCR reactions to verify gene expression. Elongation factor $1 \alpha$ of $N$. vitripennis (NvEF-1 $\alpha$ ) was used as a reference gene. Primers that were used to amplify the common region of NvDsx in all male splice-variants were designed in Geneious 10.0.9. Detailed primer sequences are listed in Table S1.

\section{Mating trials}

In the mating trials, non-injected (Non-i) females were presented with Non-i males $(n=40)$, gfp-i males $(n=40)$, or $N v D s x-i$ males $(n=60)$. One- to two-day-old virgin males and females for the mating trials were separated at black pupal stage by sex-specific traits such as forewing size and presence/absence of the ovipositor, and were collected in separate glass vials $(7.5 \mathrm{~cm}$ length, one $\mathrm{cm}$ diameter) plugged with cotton until eclosion. Each wasp was used only once. A virgin female was transferred into a glass vial ( $7.5 \mathrm{~cm}$ length, one $\mathrm{cm}$ diameter) that contained a male, and the wasps were brought in contact at the bottom by quickly flicking the vial. Observations started immediately after transferring the female. The durations of four distinctive elements of the mating process were recorded: (1) searching time, which is the time from the start of the observation until the male mounts the female; (2) mounting time, the 
duration of the male mounting the female; (3) copulation time, the duration of the copulation; (4) postcopulation time, the duration of the male remounting the female after copulation until the male dismounts. Recordings were excluded if mounting did not occur within five minutes. Mating success was pronounced positive if all four mating steps were finalized in a single mating trial and was used to calculate the mating success rate.

\section{Y-tube olfactometer bioassay}

The responses of virgin females to the odours of differently treated conspecifics were tested in a glass still-air Y-tube olfactometer $(0.9 \mathrm{~cm}$ diameter), consisting of an $8.5 \mathrm{~cm}$ stem and two five $\mathrm{cm}$ arms. Prior to the tests, ten wasps of either treatment were kept in five $\mathrm{ml}$ transparent pipette tips that were sealed at both ends with parafilm. Wasp odours were allowed to accumulate in the pipette tips by keeping the wasps inside the pipette tips for two days under rearing conditions. Afterwards, the bottom parafilm was replaced by a piece of fibre mesh, and pipette tips were mounted to the arms of the Y-tube olfactometer. Prior to each experiment, odours were allowed to diffuse for five minutes from the pipette tips into the arms of the Y-tube olfactometer. Tests started once a glass vial containing a one- to two-day-old virgin female was connected to the stem of Y-tube olfactometer. Tested females were given three minutes to enter the stem of the Y-tube olfactometer and another three minutes to make a decision. Replicates were excluded when females did not enter the stem of the Y-olfactometer in three minutes. A decision was recorded, if a female entered one arm of the olfactometer and stayed less than one $\mathrm{cm}$ away from the respective mesh for one minute. Any other cases were recorded as "no choice". After each test, the Y-olfactometer was turned by $180^{\circ}$ to compensate for any unforeseen asymmetry bias. The Y-tube olfactometer was replaced by a clean one after five tests and pipette tips with wasps were replaced by new ones after 10 tests. Each responding female was tested only once and tested female was gently brushed out of the Y-tube. Tested combinations of two choices were between: 10 Non-i females and 10 Non-i males $(n=20), 10$ Non-i females and 10 gfp-i males $(n=25), 10$ Non-i females and $10 N v D s x-i$ males ( $n=25), 10$ Non-i males and $10 \mathrm{gfp}$-i males ( $\mathrm{n}=40)$, and 10 Non-i males and $10 \mathrm{NvDsx}$-i males $(n=40)$.

7. Behavioural experiments with dead wasps 
Experiments were performed in a round bioassay chamber (10 $\mathrm{mm}$ diameter $\times 3 \mathrm{~mm}$ high) made from acrylic glass and covered by a cover slip (68). Behaviours were observed by using a stereo microscope with illumination from a microscope light, and recorded using The Observer XT software (Noldus Information Technology, Wageningen, The Netherlands). Non-i males were exposed to differently treated, freeze-killed one- to two-day-old cadavers (dummies) for five minutes. For each test, the time was recorded that the males spent mounting the dummy, whether these males tried to copulate with the dummy and, if so, the time that these copulation attempts lasted. In the first experiment, we tested the responses of Non-i males $(n=25)$ to untreated male gfp-i and NvDsx-i dummies as well as to male $N v D s x-i$ dummies treated with one $\mu \mathrm{l}$ dichloromethane (DCM, control) or one $\mu \mathrm{l}$ of a solution of Z9C31 in DCM (30 ng/ $\mu \mathrm{l})$. In the second experiment, we tested the responses of Non-i males $(n=20)$ to untreated Non-i male and female dummies as well as to Non-i female dummies treated with one $\mu$ I DCM (control) or one $\mu \mathrm{l}$ of a solution of Z9C31 in DCM (30 ng/ $\mu \mathrm{l})$. Z9C31 was synthesized based on SaulGershenz and Millar (2006). The dose of Z9C31 was based on the amount that was found in the CHC extracts of gfp-i males (see results). In all treatments that used DCM, the solvent was allowed to evaporate for one minute before the start of the bioassays.

\section{Chemical analyses}

For $\mathrm{CHC}$ analyses, individual freeze-killed two-day-old $g f p$-i and NvDsx-i males $(\mathrm{n}=10)$ were extracted for 30 minutes with $15 \mu \mathrm{l}$ hexane containing $10 \mathrm{ng} / \mu \mathrm{l}$ tetracosane as an internal standard. For analysis of the volatile sex attractant pheromone (HDL), dissected abdomens of individual freeze-killed two-dayold gfp-i and NvDsx-i males $(\mathrm{n}=10)$ were extracted with $30 \mu \mathrm{l}$ of DCM containing $10 \mathrm{ng} / \mu \mathrm{l}$ methyl undecanoate as an internal standard. Aliquots of two $\mu$ l of extracts (HDL analyses: one $\mu$ ) were analysed on a Shimadzu QP2010 Plus GC/MS system equipped with a non-polar BPX5 capillary column (60 m x $0.25 \mathrm{~mm}$ inner diameter, $0.25 \mu \mathrm{m}$ film thickness; SGE Analytical Science Europe, Milton Keynes, UK). Samples were injected at $300^{\circ} \mathrm{C}$ in splitless mode using an AOC $20 \mathrm{i}$ auto sampler. Helium was used as carrier gas at a linear velocity of $30 \mathrm{~cm} \mathrm{~s}^{-1}\left(\mathrm{HDL}: 37.8 \mathrm{~cm} \mathrm{~s}^{-1}\right)$. The initial oven temperature of $150^{\circ} \mathrm{C}$ (HDL: $80^{\circ} \mathrm{C}$ ) was increased at $2^{\circ} \mathrm{C} \mathrm{min}^{-1}\left(\mathrm{HDL}: 5^{\circ} \mathrm{C} \mathrm{min}^{-1}\right)$ to $300^{\circ} \mathrm{C}\left(\mathrm{HDL}: 280^{\circ} \mathrm{C}\right)$ and held for 30 minutes The mass spectrometer was operated in the electron ionization mode at $70 \mathrm{eV}$ and the mass range was $\mathrm{m} / \mathrm{z}$ 35-600. Identifications of compounds were done by comparing mass spectra and linear retention indices with those of authentic reference chemicals (HDL-RR, HDL-RS, 4MQ, Z9C31) or previously 
published identifications of specific CHC in this species (13) (Z7C31, Z9C33, Z7C33). Quantification of alkenes and HDL was done by comparing the peak areas of the analytes to those of the respective internal standards.

\section{Data analysis}

qPCR data was first imported to LinRegPCR software (LinRegPCR, 2017.1.0.0, HFRC, Amsterdam, The Netherlands). After baseline correction, the initial number of templates (N0) was calculated based on averaging PCR efficiency in each amplicon. Relative expression levels of target genes were obtained by dividing the NO value of target genes by the NO value of the reference gene. Statistical analysis of QPCR and mating behaviour data was performed in R (70) using general linear models (GLM) with gamma distribution and Tukey's Honestly Significant Difference (HSD) test for post-hoc comparisons. Mating success rates were compared by Bonferroni-corrected multiple $2 \times 2 \mathrm{Chi}^{2}$ tests. Y-tube olfactometer data were analysed in R using GLM with binominal distribution with cloglog link function and HSD for post-hoc comparisons. The amount of alkenes in the CHC extracts and HDL and 4MQ in the abdomen extracts of $g f p-i$ and NvDsx-i males were compared by Mann-Whitney U-tests. In tests with dummies, the total mounting times and the durations of copulation attempts were compared by a Kruskal-Wallis H-test followed by Bonferroni-corrected multiple Mann-Whitney U-tests for pairwise comparisons. The percentages of males showing copulation attempts were compared by Bonferronicorrected multiple $2 \times 2 \mathrm{Chi}^{2}$ tests.

\section{Data availability}

The datasets generated during and/or analysed during the current study are available in the figshare repository, [10.6084/m9.figshare.14572671].

\section{Author contributions}

Y.W. generated the dsRNA. Y.W and W.S. performed microinjections. W.S. conducted mating behaviour bioassays and Y-tube olfactometer experiments. Chemical analyses were carried out by J.R. Bioassays with dummies were performed by S.F. J.M. synthesized the Z9C31. Y.W. and J.R. contributed to the data analysis. Y.W., E.C.V. and J.R. contributed to the design of the work. Y.W. drafted the manuscript. E.C.V., J.R., and J.M. revised the draft manuscript. 


\section{Competing interests}

The authors declare no competing interests.

\section{Acknowledgment}

We thank Ray Owens for providing the vector pOPINEneo-3C-GFP to support this research.

\section{References}

1. W. L. Roelofs, A. P. Rooney, Molecular genetics and evolution of pheromone biosynthesis in Lepidoptera. Proc. Natl. Acad. Sci. 100, 9179-84 (2003).

2. S. Lebreton, et al., A Drosophila female pheromone elicits species-specific long-range attraction via an olfactory channel with dual specificity for sex and food. BMC Biol. 15, 1-14 (2017).

3. T. Chuman, P. J. Landolt, R. R. Heath, J. H. Tumlinson, Isolation, identification, and synthesis of male-produced sex pheromone of papaya fruit fly, Toxotrypana curvicauda Gerstaecker (Diptera: Tephritidae). J. Chem. Ecol. 13, 1979-1992 (1987).

4. J. Chemnitz, P. C. Jentschke, M. Ayasse, S. Steiger, Beyond species recognition: somatic state affects long-distance sex pheromone communication. Proc. R. Soc. B Biol. Sci. 282, 20150832 (2015).

5. J. Ruther, M. Matschke, L.-A. Garbe, S. Steiner, Quantity matters: male sex pheromone signals mate quality in the parasitic wasp Nasonia vitripennis. Proceedings. Biol. Sci. 276, 3303-3310 (2009).

6. J. Buellesbach, et al., Cuticular hydrocarbon divergence in the jewel wasp Nasonia: evolutionary shifts in chemical communication channels? J. Evol. Biol. 26, 2467-2478 (2013).

7. S. Geiselhardt, T. Otte, M. Hilker, The role of cuticular hydrocarbons in male mating behavior of the mustard leaf beetle, Phaedon cochleariae (F.). J. Chem. Ecol. 35, 1162-1171 (2009).

8. M. D. Ginzel, "Hydrocarbons as contact pheromones of longhorned beetles (Coleoptera: Cerambycidae)" in Insect Hydrocarbons: Biology, Biochemistry, and Chemical Ecology, 
(Cambridge University Press, Cambridge, UK., 2010), pp. 375-389.

9. P. J. Silk, K. Ryall, D. Barry Lyons, J. Sweeney, J. Wu, A contact sex pheromone component of the emerald ash borer Agrilus planipennis Fairmaire (Coleoptera: Buprestidae).

Naturwissenschaften 96, 601-608 (2009).

10. S. Jungwirth, J. Ruther, T. Pokorny, Similar is not the same - mate recognition in a parasitoid wasp. Front. Ecol. Evol. 9, 174 (2021).

11. M. Page, L. J. Nelson, M. I. Haverty, G. J. Blomquist, Cuticular hydrocarbons of eight species of north american cone beetles, Conophthorus hopkins. J. Chem. Ecol. 16, 1173-1198 (1990).

12. J. Ruther, "Novel insights into pheromone-mediated communication in parasitic hymenopterans" in Chemical Ecology of Insect Parasitoids, E. Wajnberg, S. Colazza, Eds. (John Wiley \& Sons, Ltd, 2013), pp. 112-144.

13. S. Steiner, N. Hermann, J. Ruther, Characterization of a female-produced courtship pheromone in the parasitoid Nasonia vitripennis. J. Chem. Ecol. 32, 1687-1702 (2006).

14. M. D. Ginzel, G. J. Blomquist, J. G. Millar, L. M. Hanks, Role of contact pheromones in mate recognition in Xylotrechus colonus. J. Chem. Ecol. 29, 533-545 (2003).

15. E. G. Uebel, P. E. Sonnet, R. W. Miller, M. Beroza, Sex pheromone of the face fly, Musca autumnalis De Geer (Diptera: Muscidae). J. Chem. Ecol. 1, 195-202 (1975).

16. J. F. Ferveur, G. Sureau, Simultaneous influence on male courtship of stimulatory and inhibitory pheromones produced by live sex-mosaic Drosophila melanogaster. Proc. R. Soc. B Biol. Sci. 263, 967-973 (1996).

17. D. A. Carlson, et al., Sex attractant pheromone of the house fly: isolation, identification and synthesis. Science 174, 76-78 (1971).

18. D. Bopp, G. Saccone, M. Beye, Sex determination in insects: variations on a common theme. Sex. Dev. 8, 20-28 (2014).

19. A. S. Wilkins, Moving up the hierarchy: a hypothesis on the evolution of a genetic sex 
determination pathway. Bioessays 17, 71-77 (1995).

20. X. Chen, et al., Disruption of sex-specific doublesex exons results in male- and female-specific defects in the black cutworm, Agrotis ipsilon. Pest Manag. Sci. 75, 1697-1706 (2019).

21. Y. Ito, et al., The role of doublesex in the evolution of exaggerated horns in the Japanese rhinoceros beetle. EMBO Rep. 14, 561-567 (2013).

22. S. Mine, M. Sumitani, F. Aoki, M. Hatakeyama, M. G. Suzuki, Identification and functional characterization of the sex-determining gene doublesex in the sawfly, Athalia rosae (Hymenoptera: Tenthredinidae). Appl. Entomol. Zool. 52, 497-509 (2017).

23. G. Rice, O. Barmina, K. Hu, A. Kopp, Evolving doublesex expression correlates with the origin and diversification of male sexual ornaments in the Drosophila immigrans species group. Evol. Dev. 20, 78-88 (2018).

24. J. N. Shukla, S. R. Palli, Doublesex target genes in the red flour beetle, Tribolium castaneum. Sci. Rep. 2, 1-10 (2012).

25. J. Xu, et al., Sexually dimorphic traits in the silkworm, Bombyx mori, are regulated by doublesex. Insect Biochem. Mol. Biol. 80, 42-51 (2017).

26. T. M. Williams, et al., The regulation and evolution of a genetic switch controlling sexually dimorphic traits in Drosophila. Cell 134, 610-623 (2008).

27. E. Demir, B. J. Dickson, fruitless splicing specifies male courtship behavior in Drosophila. Cell 121, 785-794 (2005).

28. L. C. Ryner, et al., Control of male sexual behavior and sexual orientation in Drosophila by the fruitless gene. Cell 87, 1079-1089 (1996).

29. Y. Pan, C. C. Robinett, B. S. Baker, Turning males on: activation of male courtship behavior in Drosophila melanogaster. PLoS One 6, e21144 (2011).

30. E. J. Rideout, J.-C. Billeter, S. F. Goodwin, The sex-determination genes fruitless and doublesex specify a neural substrate required for courtship song. Curr. Biol. 17, 1473-1478 
(2007).

31. E. J. Rideout, A. J. Dornan, M. C. Neville, S. Eadie, S. F. Goodwin, Control of sexual differentiation and behavior by the doublesex gene in Drosophila melanogaster. Nat. Neurosci. 13, 458-466 (2010).

32. O. M. Beckers, T. Kijimoto, A. P. Moczek, doublesex alters aggressiveness as a function of social context and sex in the polyphenic beetle Onthophagus taurus. Anim. Behav. 132, $261-$ 269 (2017).

33. A. R. Whiting, The biology of the parasitic wasp Mormoniella vitripennis = Nasonia brevicornis (Walker). Q. Rev. Biol. 42, 333-406 (1967).

34. E. C. Verhulst, et al., A new component of the Nasonia sex determining cascade is maternally silenced and regulates transformer expression. PLoS One 8, e63618 (2013).

35. L. van de Zande, E. C. Verhulst, Genomic imprinting and maternal effect genes in haplodiploid sex determination. Sex. Dev. 8, 74-82 (2014).

36. E. C. Verhulst, L. W. Beukeboom, L. van de Zande, Maternal control of haplodiploid sex determination in the wasp Nasonia. Science 328, 620-623 (2010).

37. Y. Zou, E. Geuverink, L. W. Beukeboom, E. C. Verhulst, L. van de Zande, A chimeric gene paternally instructs female sex determination in the haplodiploid wasp Nasonia. Science $\mathbf{3 7 0}$, $1115-1118$ (2020).

38. Y. Wang, et al., Sexually dimorphic traits and male-specific differentiation are actively regulated by Doublesex during specific developmental windows in Nasonia vitripennis. bioRxiv, https://doi.org/10.1101/2020.04.19.048553 (2020).

39. M. M. Mair, J. Ruther, Chemical ecology of the parasitoid wasp genus Nasonia (Hymenoptera, Pteromalidae). Front. Ecol. Evol. 7, 184 (2019).

40. J. Ruther, L. M. Stahl, S. Steiner, L. A. Garbe, T. Tolasch, A male sex pheromone in a parasitic wasp and control of the behavioral response by the female's mating status. J. Exp. Biol. 210, 2163-2169 (2007). 
41. J. Ruther, S. Steiner, L.-A. Garbe, 4-methylquinazoline is a minor component of the male sex pheromone in Nasonia vitripennis. J. Chem. Ecol. 34, 99-102 (2008).

42. M. Abdel-Latief, L. A. Garbe, M. Koch, J. Ruther, An epoxide hydrolase involved in the biosynthesis of an insect sex attractant and its use to localize the production site. Proc. Natl. Acad. Sci. 105, 8914-8919 (2008).

43. S. Steiner, J. Ruther, Mechanism and behavioral context of male sex pheromone release in Nasonia vitripennis. J. Chem. Ecol. 35, 416-421 (2009).

44. M. M. Mair, J. Ruther, Territoriality and behavioural strategies at the natal host patch differ in two microsympatric Nasonia species. Anim. Behav. 143, 113-129 (2018).

45. M. Lenschow, et al., The post-mating switch in the pheromone response of Nasonia females is mediated by dopamine and can be reversed by appetitive learning. Front. Behav. Neurosci. 12, 14 (2018).

46. J. Buellesbach, S. G. Vetter, T. Schmitt, Differences in the reliance on cuticular hydrocarbons as sexual signaling and species discrimination cues in parasitoid wasps. Front. Zool. 15, 22 (2018).

47. O. Niehuis, J. Büllesbach, A. K. Judson, T. Schmitt, J. Gadau, Genetics of cuticular hydrocarbon differences between males of the parasitoid wasps Nasonia giraulti and Nasonia vitripennis. Heredity 107, 61-70 (2011).

48. R. Barrass, The Courtship Behaviour of Mormoniella vitripennis Walk. (Hymenoptera, Pteromalidae). Behaviour 15, 185-209 (1960).

49. J. van den Assem, J. H. Werren, A comparison of the courtship and mating behavior of three species of Nasonia (Hymenoptera: Pteromalidae). J. Insect Behav. 7, 53-66 (1994).

50. J. van den Assem, P. Simbolotti, F. Jachmann, Courtship behaviour of Nasonia vitripennis (Hymenoptera, Pteromalidae): Some qualitative, experimental evidence for the role of pheromones. Behaviour 75, 301-307 (1980).

51. J. Ruther, J. McCaw, L. Böcher, D. Pothmann, I. Putz, Pheromone diversification and age- 
dependent behavioural plasticity decrease interspecific mating costs in Nasonia. PLoS One $\mathbf{9}$, e89214 (2014).

52. B. Blaul, J. Ruther, How parasitoid females produce sexy sons: a causal link between oviposition preference, dietary lipids and mate choice in Nasonia. Proc. R. Soc. B Biol. Sci. 278, 3286-3293 (2011).

53. J. Ruther, A. Bruckmann, J. Hofferberth, "Pheromone biosynthesis in Nasonia" in Insect Pheromone Biochemistry and Molecular Biology, second, (Elsevier Scientific, 2021), pp. 237267.

54. J.-M. Jallon, G. Lauge, L. Orssaud, C. Antony, Female pheromones in Drosophila melanogaster are controlled by the doublesex locus. Genet. Res. 51, 17-22 (1988).

55. J. A. Waterbury, L. L. Jackson, P. Schedl, Analysis of the Doublesex female protein in Drosophila melanogaster: role in sexual differentiation and behavior and dependence on intersex. Genetics 152, 1653-1667 (1999).

56. F. Lacaille, et al., An inhibitory sex pheromone tastes bitter for Drosophila males. PLoS One 2, e661 (2007).

57. D. A. Carlson, C. J. Geden, U. R. Bernier, Identification of pupal exuviae of Nasonia vitripennis and Muscidifurax raptorellus parasitoids using cuticular hydrocarbons. Biol. Control 15, 97-106 (1999).

58. J.-M. Jallon, J. R. David, Variations in cuticular hydrocarbons among the eight species of the Drosophila melanogaster subgroup. Evolution 41, 294-302 (1987).

59. T. Chertemps, L. Duportets, C. Labeur, M. Ueyama, C. Wicker-Thomas, A female-specific desaturase gene responsible for diene hydrocarbon biosynthesis and courtship behaviour in Drosophila melanogaster. Insect Mol. Biol. 15, 465-473 (2006).

60. T. R. Shirangi, H. D. Dufour, T. M. Williams, S. B. Carroll, Rapid evolution of sex pheromoneproducing enzyme expression in Drosophila. PLoS Biol. 7, e1000168 (2009).

61. M. D. Drapeau, J. H. Werren, Differences in mating behaviour and sex ratio between three 
sibling species of Nasonia. Evol. Ecol. Res. 1, 223-224 (1999).

62. J. H. Werren, et al., Functional and evolutionary insights from the genomes of three parastoid Nasonia species. Science 327, 343-348 (2010).

63. T. R. R. Shirangi, A. M. M. Wong, J. W. W. Truman, D. L. L. Stern, Doublesex regulates the connectivity of a neural circuit controlling Drosophila male courtship song. Dev. Cell 37, 533544 (2016).

64. J. Ruther, K. Thal, B. Blaul, S. Steiner, Behavioural switch in the sex pheromone response of Nasonia vitripennis females is linked to receptivity signalling. Anim. Behav. 80, 1035-1040 (2010).

65. J. Ruther, T. Hammerl, An oral male courtship pheromone terminates the response of Nasonia vitripennis females to the male-produced sex attractant. J. Chem. Ecol. 40, 56-62 (2014).

66. I. Mikó, A. Deans, The mandibular gland in Nasonia vitripennis (Hymenoptera: Pteromalidae). bioRxiv, 006569 (2014).

67. J. A. Lynch, C. Desplan, A method for parental RNA interference in the wasp Nasonia vitripennis. Nat. Protoc. 1, 486-494 (2006).

68. J. Ruther, M. Homann, J. L. M. Steidle, Female-derived sex pheromone mediates courtship behaviour in the parasitoid Lariophagus distinguendus. Entomol. Exp. Appl. 96, 265-274 (2000).

69. L. S. Saul-Gershenz, J. G. Millar, Phoretic nest parasites use sexual deception to obtain transport to their host's nest. Proc. Natl. Acad. Sci. 103, 14039-14044 (2006).

70. R core Team, R: A Language and Environment for Statistical Computing. R Foundation for Statistical Computing, Vienna, Austria (2018). 
Figure and figure legends

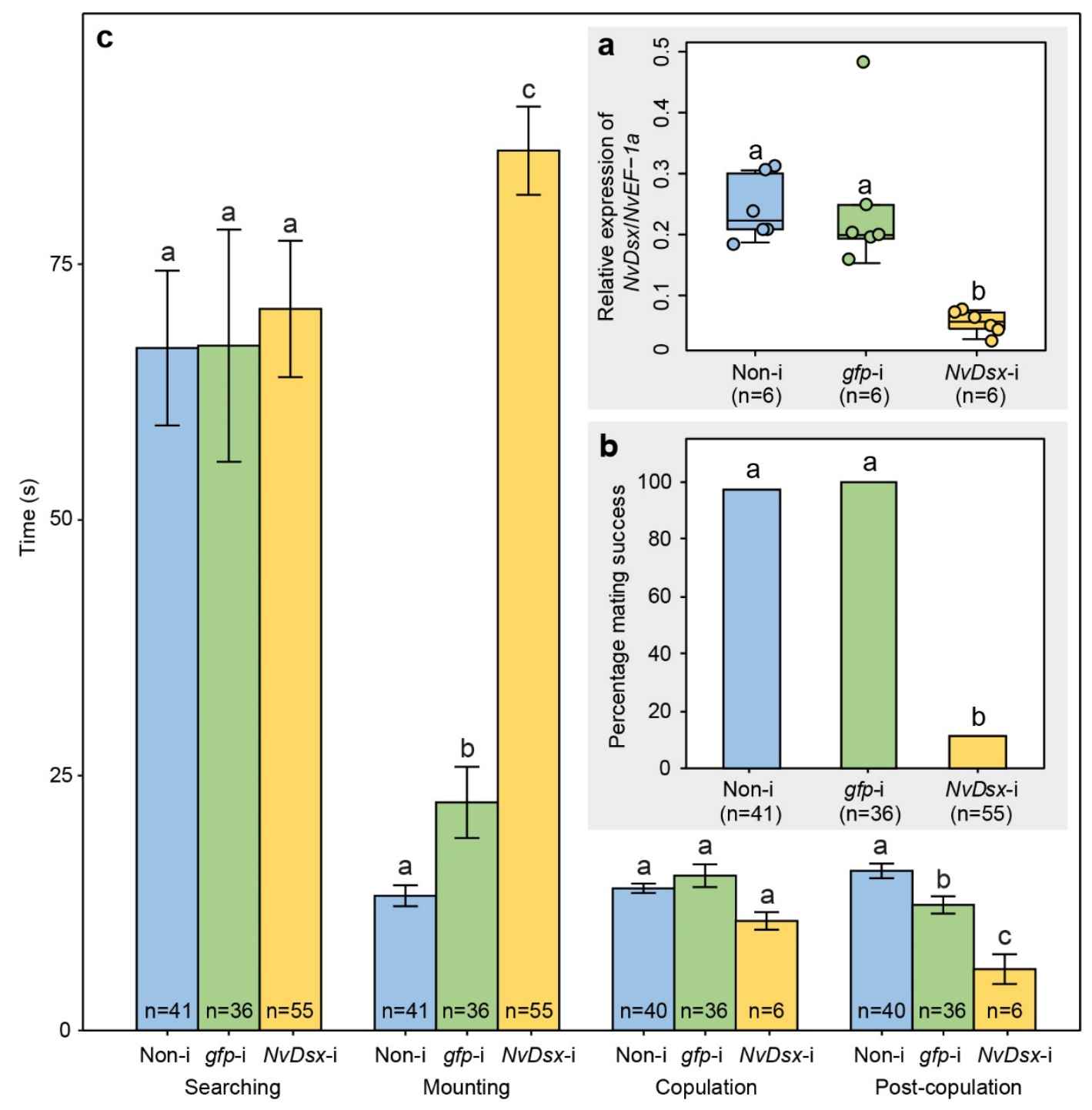

Fig. 1 Effects of NvDsx knockdown on NvDsx expression and mating behaviour of $N$. vitripennis

males. a Relative expression of $N v D s x / N v E F-1 a$ in Non-i, gfp-i and NvDsx-i males. Box-and-whisker plot shows median values (horizontal line), 25-75\% quartiles (box), maximum/minimum range (whiskers) and individual data points. b Percentage mating success of Non-i, gfp-i and NvDsx-i males in mating trials with virgin females. c Duration of selected behavioural elements (means $\pm \mathrm{SE}$ ) of the $N$. vitripennis mating sequence of Non-i, gfp-i, and NvDsx-i males in mating trials with virgin females. Different lowercase letters indicate significant differences at $P<0.05$ (a, c: GLM, b: Bonferroni-corrected multiple Chi tests). 

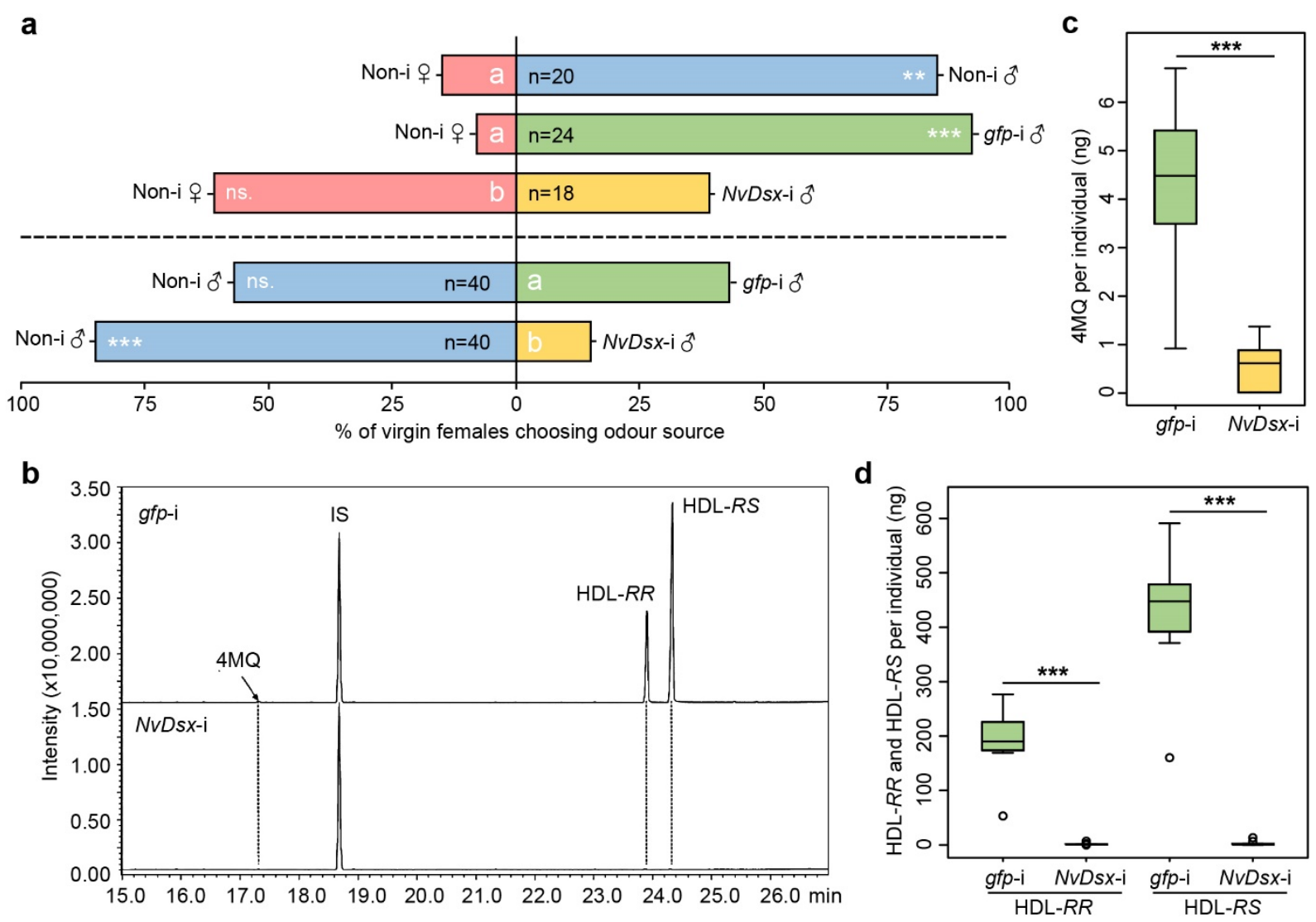

Fig. 2 Response of virgin females to the odours of differently treated conspecifics in a Y-tube olfactometer, and chemical analysis of the abdominal sex attractant pheromone in $g f p-i$ and

NvDsx-i males. a Percentage of virgin females choosing between the odour of ten live Non-i females, Non-i males, gfp-i, or NvDsx-i males. The treatments tested in each experiment are given on each side of the bars. Statistical analyses were conducted in two groups separated by the dashed line. Letters indicate significant differences at $P<0.05$ (GLM) and deviations from equality are indicated by asterisks, ns. refers to not significant $(P>0.05),{ }^{* *}$ refers to $P<0.01,{ }^{* * *}$ refers to $P<0.001$ (GLM). b Total ion current chromatograms of abdominal extracts from $g f p-i$ and NvDsx-i males (IS = internal standard, $10 \mathrm{ng}$ methyl undecanoate, HDL-RR and HDL- $R S=(4 R, 5 R)$ - and $(4 R, 5 S)$-5-hydroxy-4-decanolide, $4 \mathrm{MQ}=4$ methylquinazoline). Quantification of $\mathbf{c} 4 \mathrm{MQ}$, and $\mathbf{d} \mathrm{HDL}-R R$ and HDL-RS in abdominal extracts from gfp-i and NvDsx-i males. Box-and-whisker plots show median (horizontal line), 25-75 \% quartiles (box), maximum/minimum range (whiskers) and outliers $\left({ }^{\circ}>1.5 \mathrm{x}\right.$ interquartile range outside the first or the third quartile). Asterisks indicate significant differences between treatments at $P<0.001$ (Mann-Whitney U-test, $n=10)$. 

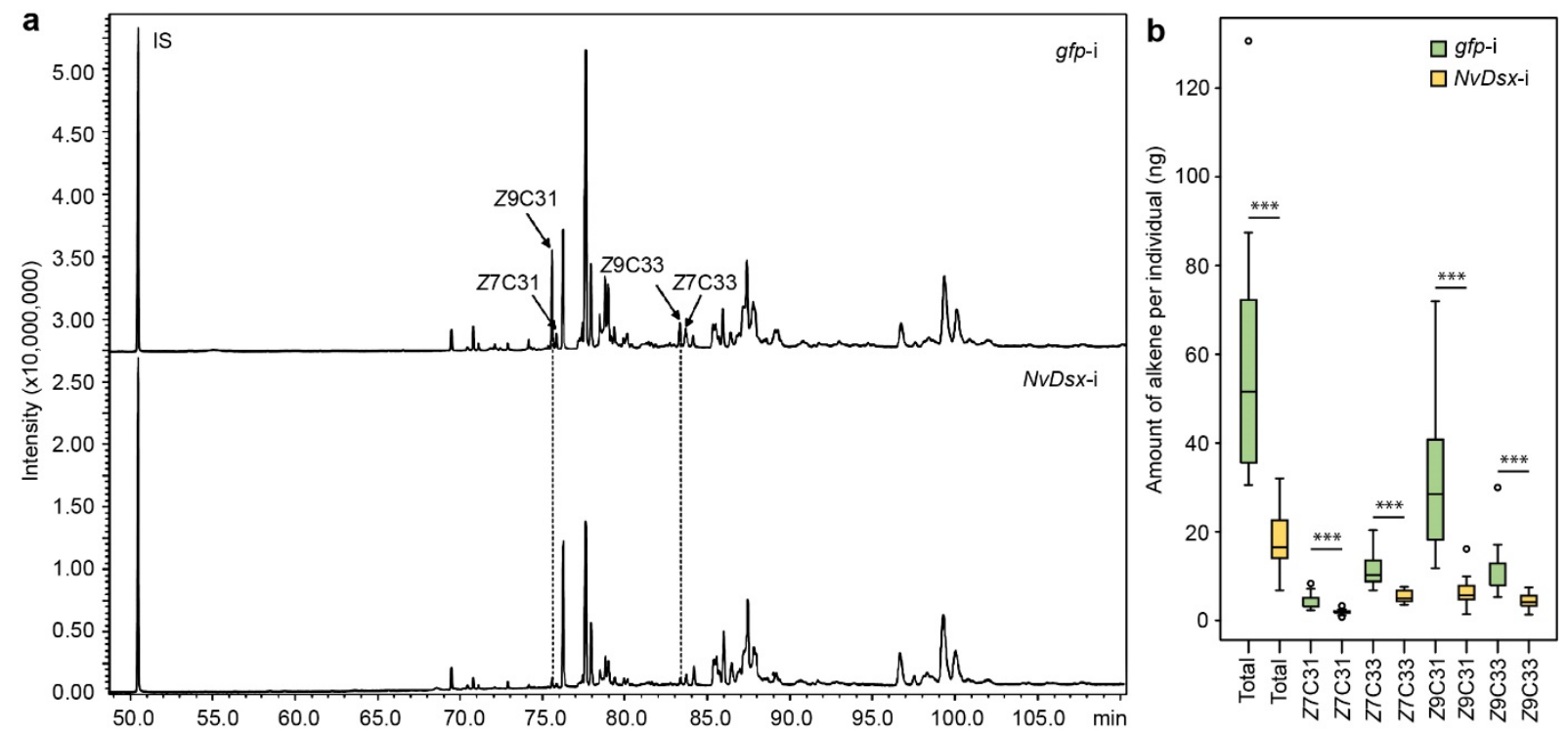

Fig. 3 Chemical analyses of cuticular hydrocarbons in NvDsx-i and gfp-i males. a Total ion chromatograms of whole-body hexane extracts from gfp-i and Nvdsx-i males. Arrows indicate peaks of the alkenes (Z)-9- and (Z)-7-hentriacontene (Z9C31 and Z7C31) as well as (Z)-9- and (Z)-7tritriacontene (Z9C33 and Z7C33). IS = internal standard (10 ng tetracosane). b Quantification of alkenes in dichloromethane extracts of abdomens of $g f p-i$ and $N v d s x-i$ males. The box-and-whisker plots show median (horizontal line), $25-75 \%$ quartiles (box), maximum/minimum range (whiskers) and outliers $\left({ }^{\circ}>1.5 \mathrm{x}\right.$ interquartile range outside the first or third quartile). Asterisks indicate significant differences between treatments at $P<0.001$ (Mann-Whitney $U$-test, $\mathrm{n}=10$ ). 

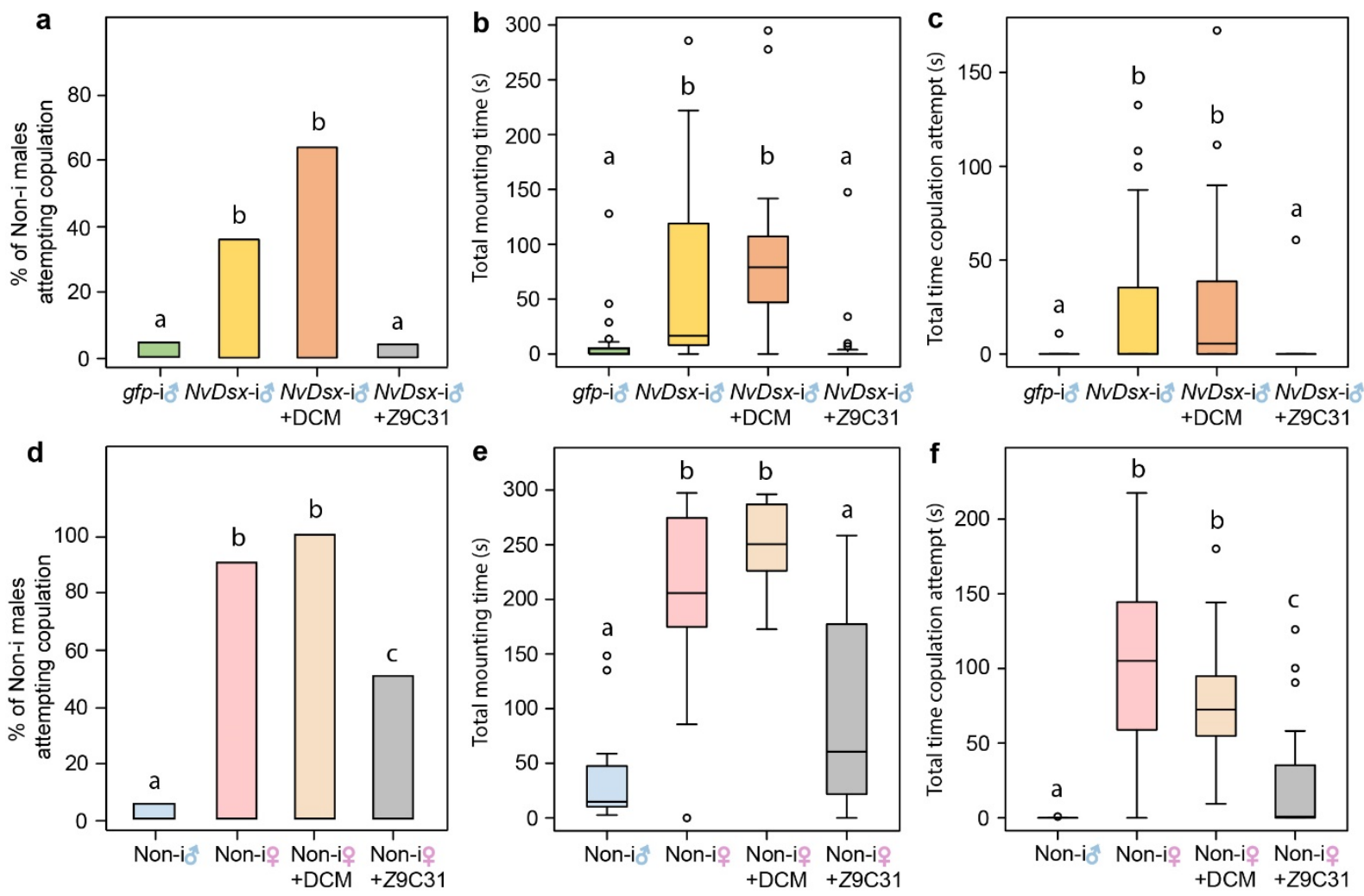

Fig. 4 Effect of synthetic Z9C31 on the courting behaviour of Non-i $N$. vitripennis males.

Responding Non-i males were presented with dead conspecifics (dummies) for a five minutes observation period. Dummies were either differently treated gfp-i or $N v D s x-i$ males $(a-c, n=25)$ or Non-i wasps of either sex $(d-f, n=20)$. Tested dummies were either untreated, treated with $30 \mathrm{ng}$ of $(Z)-9-$ hentriacontene (Z9C31) dissolved in dichloromethane (DCM), or with pure DCM. Shown is a, d the percentage of Non-i males trying to copulate with the dummy, $\mathbf{b}, \mathbf{e}$ the total mounting time, and $\mathbf{c}, \mathbf{f}$ the duration of copulation attempts. Box-and-whisker plots (b, c, e, and f) show median (horizontal line), 25$75 \%$ quartiles (box), maximum/minimum range (whiskers) and outliers $\left({ }^{\circ}>1.5 \mathrm{x}\right.$ interquartile range outside the first or third quartile). Letters indicate significant differences at $P<0.05$ (data analysis by Kruskal-Wallis $\mathrm{H}$-test followed by Bonferroni-corrected multiple Mann-Whitney $\mathrm{U}$-tests $(\mathbf{b}, \mathbf{c}, \mathbf{e}$, and $\mathbf{f})$ or by Bonferroni-corrected multiple $2 \times 2 \mathrm{Chi}^{2}$-tests ( $\mathbf{a}$ and $\mathbf{d}$ ). 